\title{
Biomonitorización de plaguicidas: ¿Una necesidad del país?
}

\author{
JUAN CARLOS RÍOS B. ${ }^{1,2}$, SANDRA SOLARI G. ${ }^{1,2}$
}

\section{Biomonitoring pesticides: A national need?}

The word 'biomarker' is being used more frequently during the last decade. Currently, a great amount of research is directed to find toxicological biomarkers for several different chemical substances, since persons are more exposed now than decades before. For this reason, it is important to perform a biomonitoring of such substances in order to assess the possibility that low quantities could cause health problems. Obtaining toxicological values through biomonitoring is also useful to control the exposure. The markers of poisoning by chemicals available worldwide are numerous, but unfortunately in Chile only a few are being used. It is essential to have more toxicology and environmental laboratories to test the levels of exposure of our population.

(Rev Med Chile 2010; 138: 515-518).

Key words: Biological markers; Clinical Laboratories; Pesticides; Toxicology.

\section{RESUMEN}

El término biomarcador ha tenido un uso explosivo en la última década y el área de la toxicología no ha sido la excepción. En la actualidad se busca obtener el mayor número de biomarcadores toxicológicos de sustancias químicas a las que un ser humano puede estar expuesto. La biomonitorización de los agentes químicos es fundamental para conocer en qué concentraciones estas sustancias pueden ocasionar algún problema de salud en la población y también es útil para regular la exposición a ellos. A nivel mundial, son numerosos los marcadores disponibles de intoxicación por sustancias químicas, pero en Chile son pocos los que se utilizan en forma amplia. Dado lo anterior, es fundamental implementar laboratorios toxicológicos y ambientales capaces de realizar la biomonitorización de plaguicidas en forma exhaustiva $y$ confiable.

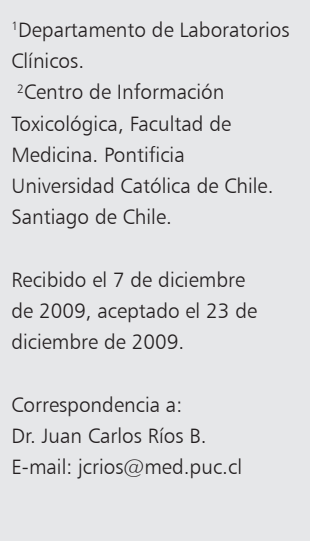

\section{DE LA VIDA REAL}

Un grupo de trabajadores manifiesta su preocupación en la empresa donde trabajan, debido a que a uno de sus compañeros se le diagnosticó recientemente una intoxicación aguda por plaguicidas. Ellos trabajan con diferentes tipos de compuestos, pero desconocen cuál fue el producto implicado en esta intoxicación. La empresa envía a todo el grupo de empleados a realizarse un examen que mide acetilcolinesterasa en glóbulos rojos. Cuando llega su resultado, se les informa que está dentro de rangos normales.

¿Se puede descartar la exposición a plaguicidas con esta medición?

¿Es posible evaluar el riesgo de exposición a plaguicidas sólo con este examen? 


\section{Biomarcadores}

Es difícil determinar cuándo y dónde se originaron los términos "marcador biológico" y "biomarcador". La búsqueda en la literatura indica que primero aparecieron en publicaciones a finales de 1960 y que a mediados de la década de 1980 ocurrió un crecimiento exponencial en el uso de estos términos. Como resultado de esta generalización los términos tienen varios significados, con algunas diferencias sutiles y otras mayores dependiendo de la subespecialidad particular donde sean usados. Una definición general de "biomarcador" podría ser "una medición en un sistema biológico como indicador de exposición, efecto, susceptibilidad o enfermedad clínica"1. Cabe destacar que en las últimas dos décadas se ha visto una gran expansión de tecnologías y aplicaciones que involucran este tipo de medidas.

Mientras los biomarcadores para enfermedades clínicas tienen una larga historia, no ocurre lo mismo para la exposición y detección de efectos adversos de agentes químicos (biomarcadores toxicológicos), a pesar de que se han hecho esfuerzos reiterados para descubrirlos y validarlos. Los biomarcadores toxicológicos tienen primariamente sus raíces en dos distintas áreas: la toxicología forense y la salud ocupacional donde, en esta última, la determinación de niveles máximos de seguridad de exposición a químicos en el lugar de trabajo, llevó a la descripción de los limites de exposición ocupacional y los índices de exposición biológica (BEIs). Los BEIs son medidas directas de xenobióticos y/o sus metabolitos en sangre u orina y son considerados como biomarcadores de exposición que reflejan el cumplimiento de los límites de exposición laboral ${ }^{1,2}$.

\section{Biomonitorización}

La biomonitorización es la evaluación de la exposición humana a sustancias químicas midiendo el compuesto original o sus metabolitos en muestras biológicas, como la sangre o la orina, donde estos niveles reflejan la cantidad de la sustancia química que realmente ha entrado en el organismo desde el medio ambiente. Los productos químicos deben ser siempre evaluados en su composición, ya que esto nos permite conocer la gravedad de los efectos sobre la salud. Cuando existe una exposición a sustancias químicas, es importante disponer de un método analítico de biomonitorización con una precisión, sensibilidad y especificidad adecuadas ${ }^{2,4}$.

Un programa de biomonitorización debiera constar de las siguientes etapas:

a) Detección y monitorización de exposiciones a las sustancias químicas;

b) Evaluación del riesgo de la exposición;

c) Desarrollo e implementación de intervenciones que reduzcan la exposición;

d) Evaluación de la efectividad de estas intervenciones ${ }^{4}$.

Es importante que las autoridades de salud conozcan la información de biomonitorización para así poder tomar las mejores decisiones que van en beneficio de la salud de las personas.

\section{Los plaguicidas}

Los plaguicidas son compuestos químicos, orgánicos o inorgánicos, o sustancias naturales que se utilizan para combatir malezas, enfermedades o plagas, potencialmente capaces de causar perjuicios en organismos vivos u objetos. Entre los plaguicidas, existen innumerables sustancias y de acuerdo a su importancia en cuanto a las intoxicaciones los grupos más relevantes son:

Organoclorados.

Organofosforados y carbamatos.

Piretrinas y piretroides.

Anticoagulantes ${ }^{3}$.

Los plaguicidas organoclorados actualmente están prohibidos en gran parte del mundo por su toxicidad y por su bioacumulación, siendo los agentes principales el DDT, el hexaclorobenzeno y el pentaclorofenol. Éstos se encuentran en altas concentraciones en productos grasos e ingresan al organismo principalmente por la ingesta de leche, subproductos de ella y pescados. Los compuestos organofosforados son utilizados principalmente como insecticidas, poseen características químicas similares entre sí y son inhibidores de la enzima acetilcolinesterasa. Alrededor del 75\% de los organofosforados son metabolizados a sustancias medibles, denominados dialquilfosfatos (Tabla 1), que no son considerados tóxicos, pero sí son marcadores de exposición a organofosforados. Los 
Biomonitorización de plaguicidas: ¿Una necesidad del país? - J.C. Ríos B. et al

Tabla 1. Plaguicidas organofosforados y sus respectivos metabolitos

\begin{tabular}{|c|c|c|c|c|c|c|}
\hline Pesticida & $\begin{array}{l}\text { Dimetil- } \\
\text { fosfato }\end{array}$ & $\begin{array}{l}\text { Dimetiltio- } \\
\text { fosfato }\end{array}$ & $\begin{array}{l}\text { Dimetilditio- } \\
\text { fosfato }\end{array}$ & $\begin{array}{l}\text { Dietil- } \\
\text { fosfato }\end{array}$ & $\begin{array}{l}\text { Dietiltio- } \\
\text { fosfato }\end{array}$ & $\begin{array}{l}\text { Dietilditio- } \\
\text { fosfato }\end{array}$ \\
\hline Azinfosmetil & * & * & * & & & \\
\hline Clorpirifos & & & & * & * & \\
\hline Diclorvos & * & & & & & \\
\hline Diazinon & & & & * & * & \\
\hline Dimetoato & * & * & * & & & \\
\hline Fenitotrion & * & * & & & & \\
\hline Malation & * & * & * & & & \\
\hline Paration & & & & * & * & \\
\hline Terbufos & & & & * & * & * \\
\hline
\end{tabular}

insecticidas carbamatos no persisten largo tiempo en el medio ambiente y tienen bajo potencial de acumulación. También son inhibidores de la enzima acetilcolinesterasa, sin embargo su unión es reversible 2 .

Otro grupo de plaguicidas ampliamente utilizados son los piretroides, compuestos sintéticos derivados de las piretrinas, sustancias de origen natural. Actualmente su uso ha sido masificado en el ambiente doméstico, edificios públicos, comerciales y en la agricultura, para el control de una gran variedad de insectos ${ }^{2,5}$.

\section{¿Qué se puede medir?}

El examen de acetilcolinesterasa es utilizado para el diagnóstico de la intoxicación por plaguicidas organofosforados y puede ser utilizado como indicador de exposición a este tipo de sustancias; sin embargo, su utilidad está limitada a exposiciones significativas. En Chile, el Decreto Supremo No 594 establece el reglamento sobre condiciones sanitarias y ambientales básicas en los lugares de trabajo y en su Título $\mathrm{V}$ determina los límites de tolerancia biológica incluyendo al lindano, el pentaclorofenol y los pesticidas organofosforados y carbamatos dentro de su legislación. Como indicador biológico para los pesticidas organofosforados y carbamatos se emplea la medición de actividad de acetilcolinesterasa, con un valor límite de tolerancia biológica del 70\% de la línea base de la persona (con lo que generalmente no se cuenta). Exposiciones leves pueden alterar el límite de tolerancia biológica, sin embargo, sólo en caso que la valoración biológica demuestre que han sido sobrepasados estos límites se obliga a iniciar acciones necesarias que eviten el daño a la salud del trabajador, derivado de las condiciones laborales. Lamentablemente, en nuestro país no se cuenta con otros biomarcadores toxicológicos ${ }^{6}$.

Actualmente, en EEUU se realiza monitorización biológica de un mayor número de pesticidas, algunos de los cuales se encuentran listados en las Tablas 1 y $2^{2,3,5}$.

\section{Conclusiones y cierre del caso}

¿Se puede descartar la exposición a plaguicidas con este biomarcador? La respuesta es no. Sólo podríamos descartar una exposición a compuestos organofosforados si el porcentaje de inhibición es mayor al 70\% de la línea base, según la legislación vigente. En cuanto a los pesticidas carbamatos su interpretación es aun más compleja debido a que la inhibición de la enzima es reversible, por lo cual su valor es muy impredecible.

¿Es posible evaluar el riesgo de exposición a plaguicidas sólo con este examen?

La determinación de la acetilcolinesterasa tiene varias aplicaciones: evaluación de la función hepática, la exposición a pesticidas organofosfo- 
Tabla 2. Metabolitos en sangre y orina de diferentes plaguicidas

\begin{tabular}{|c|c|c|}
\hline Pesticida & Metabolito en sangre & Metabolito en orina \\
\hline Hexaclorobenzeno & Hexaclorobenzeno & $\begin{array}{l}\text { Pentaclorofenol, 2,4,6 triclorofenol y 2,4,5 } \\
\text { triclorofenol }\end{array}$ \\
\hline Pentaclorofenol & & Pentaclorofenol \\
\hline DDT & $p, p^{\prime}-D D E$ & \\
\hline Carbofurano & & Carbofuranfenol \\
\hline Cis-Permetrina & & $\begin{array}{l}\text { Ácido cis-3-(2,2-Diclorovinil)-2,2-dimetilciclo- } \\
\text { propano carboxílico }\end{array}$ \\
\hline Deltametrina & & $\begin{array}{l}\text { Ácido cis-3-(2,2-Dibromovinil)-2,2-dimetilciclo- } \\
\text { propano carboxílico }\end{array}$ \\
\hline Cipermetrina, Deltametrina y Permetrina & & Ácido 3-fenoxibenzoico \\
\hline
\end{tabular}

rados y la identificación de la hipersensibilidad a succinilcolina. Por esta razón, evaluar el riesgo de exposición con este indicador inespecífico es muy complejo. Sin embargo, podemos identificar una intoxicación con este parámetro. Evaluar de forma correcta el riesgo implicaría determinar la concentración de la sustancia en el organismo.

En la actualidad es imposible para la población general no estar expuesta a cientos de sustancias químicas, y entre ellas están los plaguicidas. Estas sustancias pueden ingresar al organismo a través de los alimentos, el aire, el agua o la tierra. Es importante conocer los parámetros biológicos existentes en la población general para poder evaluar el riesgo de las exposiciones a este tipo de sustancias. Por esta razón, es fundamental contar con laboratorios de toxicología que midan estas sustancias químicas en muestras biológicas. Además, estos valores poblacionales son necesarios para que la autoridad determine las políticas públicas de protección de la salud de las personas en referencia a la exposición de plaguicidas.

\section{Referencias}

1. De Caprio A. Introduction to Toxicologic Biomarkers. En: Toxicologic biomarkers. EEUU, Taylor \& Francis Group 2006; p 1-17.

2. CDC Staff. Third National Report on Human Exposure to Environmental Chemicals. NCEH Pub. No. 05-0570. Publication date: 07/01/2005. Publisher: Centers for Disease Control and Prevention.

3. Ríos J, Paris E, Maqueira D, Mieres J, Bettini M, Cerda $\mathrm{P}$, et al. Epidemiología de las Intoxicaciones: Análisis de las llamadas efectuadas durante el año 2007 al Centro de Información Toxicológica (CITUC) de la Pontificia Universidad Católica de Chile. Cuad Med Soc (Chile) 2009; 49: 49-60.

4. Boogaard P, Money C. A proposed framework for the interpretation of biomonitoring data. Environ Health 2008; 5 (7 Suppl 1): S12.

5. Angerer J, Ewers U, Wilhelm M. Human biomonitoring: State of the art. Internat J Hygiene Environm Health 2007; 210, 201-28.

6. Decreto supremo 594 1999; Disponible en: www.minsal. cl [Consultado el 18 de noviembre de 2009]. 